\title{
O sistema CEREC na confecção de prótese parcial fixa em cerâmica: Relato de caso clínico
}

\author{
The CEREC system in the making of fixed partial prosthesis in ceramics: A clinical case report \\ El sistema CEREC en la fabricación de próteses parcial fija em cerâmica: Reporte de un caso \\ clínico
}

Recebido:15/05/2021 | Revisado: 21/05/2021 | Aceito: 23/05/2021 | Publicado: 09/06/2021

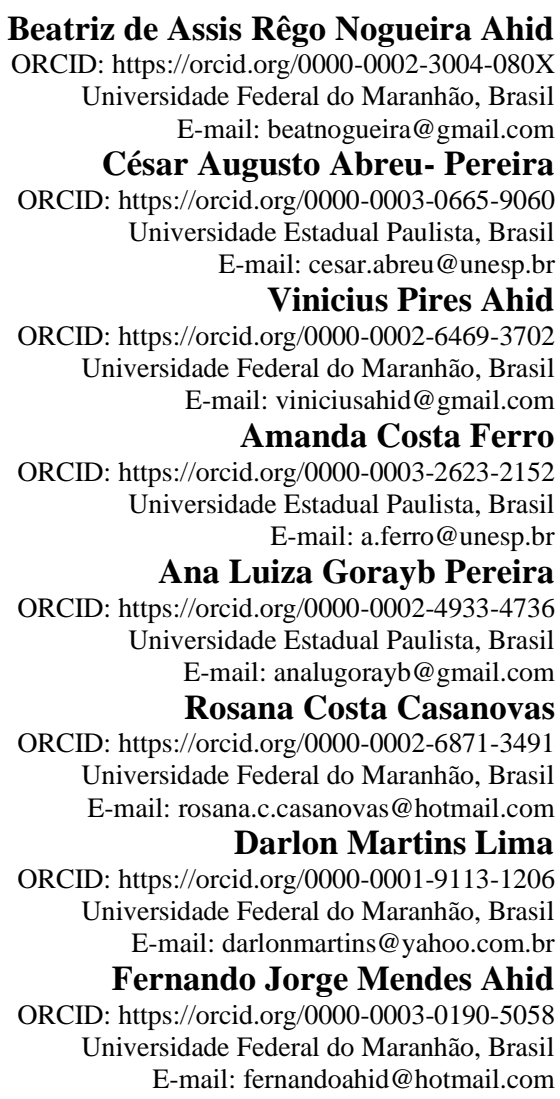

\begin{abstract}
Resumo
As restaurações indiretas são comumentes fabricadas em laboratórios de prótese especializados requerendo mais etapas, pois estes laboratórios usualmente dependem de um técnico calibrado com experiência e habilidade, além de receberem vários trabalhos de diferentes dentistas. E como resposta à dependência de cirurgiões-dentistas em relação aos laboratórios de prótese, ocorreu o surgimento da tecnologia CAD/CAM (Computer-Aided Design/Computer-Aided Manufacturing) que vem transformando a maneira de realizar restaurações indiretas. O CAD/CAM é uma evolução da informática e da engenharia para as necessidades da odontologia e aumentou dramaticamente durante a última década, graças ao rápido desenvolvimento da tecnologia de computadores digitais, por meio de escaneadora intraoral e máquina fresadora, que podem ser executados vários sistemas como o CEREC. Assim, consegue-se otimizar as consultas, apresentando excelentes resultados estéticos e longevidade clínica. Com o sistema CEREC para dentistas, toda a etapa laboratorial pode ser desenvolvida pelo próprio dentista dentro do consultório odontológico. Este artigo tem como objetivo apresentar o sistema CEREC esclarecendo seu manuseio intuitivo por meio do relato de caso clínico.
\end{abstract}

Palavras- chave: Restauração; Cad-cam; Cerâmica.

\begin{abstract}
Indirect restorations are commonly manufactured in specialized prosthesis laboratories requiring more steps because these laboratories usually rely on a calibrated technician with experience and skill, in addition to receiving various jobs from different dentists. Like response to the dependence of dental surgeons on denture laboratories, the emergence of
\end{abstract}


Computer-Aided Design (CAD / CAM) technology has been transforming the way indirect restorations are performed. $\mathrm{CAD} / \mathrm{CAM}$ is an evolution of computer science and engineering to the needs of dentistry and has increased dramatically over the last decade thanks to the rapid development of digital computer technology by means of intraoral scanner and milling machine which can be run several systems such as CEREC. Thus, it is possible to optimize the consultations, presenting excellent aesthetic results and clinical longevity. With the CEREC system for dentists, the whole laboratory stage can be developed by the dentist himself inside the dental office. This article aims to present the CEREC system clarifying its intuitive handling through the clinical case report.

Keywords: Restauration; Cad-cam; Ceramic.

\section{Resumen}

Las restauraciones indirectas se fabrican comúnmente en laboratorios de prótesis especializados que requieren más pasos porque estos laboratorios generalmente dependen de un técnico calibrado con experiencia y habilidad, además de recibir varios trabajos de diferentes dentistas. Como respuesta a la dependencia de los cirujanos dentales de los laboratorios de prótesis, la aparición de la tecnología de diseño asistido por ordenador (CAD / CAM) ha ido transformando la forma en que se realizan las restauraciones indirectas. CAD / CAM es una evolución de la informática y la ingeniería a las necesidades de la odontología y ha aumentado drásticamente durante la última década gracias al rápido desarrollo de la tecnología informática digital mediante escáner intraoral y fresadora que pueden ejecutar varios sistemas como CEREC. Así, es posible optimizar las consultas, presentando excelentes resultados estéticos y longevidad clínica. Con el sistema CEREC para dentistas, el propio dentista puede desarrollar toda la etapa del laboratorio dentro del consultorio. Este artículo tiene como objetivo presentar el sistema CEREC aclarando su manejo intuitivo a través del reporte de caso clínico.

Palabras clave: Restauración; Cad-cam; Cerámico.

\section{Introdução}

As restaurações indiretas são comumente fabricadas em laboratórios de prótese especializados, o que normalmente demanda bastante tempo, pois estes dependem de um técnico calibrado e costumam receber vários trabalhos de diferentes dentistas (Dukic et al., 2010). Tais técnicos às vezes não conseguem manter um padrão de qualidade mensurável, tornando o trabalho, mesmo com protocolo estabelecido, bastante empírico (Miyazakil et al.,2009; Poticny et al., 2010). Porém, o desenvolvimento tecnológico avança em diversas áreas do conhecimento, inclusive na odontologia. E como resposta à dependência de cirurgiões-dentistas em relação à problemática dos laboratórios de prótese, ocorreu o surgimento da tecnologia CAD/CAM que vem transformando a maneira de realizar restaurações indiretas (Hilgert, et al.,2005; Poticney et al., 2010).

O sistema CAD/CAM (Computer-Aided Design/Computer-Aided Manufacturing) é um instrumento da informática e da engenharia criado para atender às necessidades da odontologia, tendo sido bastante difundido durante a última década (Hilgert, et al.,2005), possibilitando que dispositivos cerâmicos possam ser feitos dentro das clínicas odontológicas por meio de escaneadora intraoral e máquina fresadora (Duret et al.,1988; Moreno et al., 2021). Este aparato otimiza a rotina das consultas, apresenta excelentes resultados estéticos e alta longevidade clínica (Duret et al., 1988; Keshvad et al., 2011).

A utilização desta tecnologia para confecção de restaurações foi desenvolvida por Duret em 1970, originando o Duret System que confeccionava coroas. Todavia, este sistema não obteve ampla aceitação pela dificuldade de execução e altíssimo custo (Miyazakil et al., 2009; Kim et al., 2015). O primeiro CAD/CAM comercialmente viável foi o CEREC 1 (computerassisted CERamic REConstruction) que foi executado por Mormann e Brandestini em 1983, e possibilitava a realização de restaurações indiretas em uma única sessão (Mormann et al.,2006; Fasbinder et al., 2010; Fasbinder et al., 2013).

O sistema CEREC 3, utilizado na atualidade, foi desenvolvido por equipes da Siemens e Sirona (Bensheim, Alemanha) e é indicado no processo de reabilitação oral para fabricação de próteses do tipo inlays, onlays, coroas totais anteriores e posteriores, laminados cerâmicos, pontes fixas, copings, provisórios e abutments de implantes de titânio ou zircônia utilizando softwares tridimensionais mais ilustrativos que as versões anteriores e de manipulação mais fácil e intuitiva com registro funcional dos dentes antagonistas e dos preparos dentários (Nakamura et al., 2003). Na versão CEREC 3D, o sistema funciona em 3 etapas: ferramenta de digitalização (scanner), desenho/anatomização (software) e unidade de fresagem (Beuer et al.,2008). O escaneamento se mostra como uma das principais etapas responsáveis pela precisão das peças confeccionadas, sendo realizado 
com uma câmera intraoral ou através de um scanner de bancada (Beuer et al.,2008). Este sistema apresenta 5 tipos de scanners com sistema fechado, sendo 2 intra-orais para dentistas: o CEREC Omnicam e o CEREC Bluecam. Todos são capazes de adquirir impressões visuais de um quadrante em menos de um minuto e o escaneamento dos antagonistas levam apenas alguns segundos (Sirona, 2018).

As principais diferenças clínicas entre CEREC Bluecam e Omnicam é que a primeira possui um sistema interno de detecção de movimento que as imagens são assimiladas somente quando a câmera está estática e necessita da utilização de um agente de contraste (dióxido de titânio) sobre as superfícies preparadas para que exista reflexão adequada e uniforme da luz, capaz de ser corretamente interpretada pela câmera. Em contrapartida, com a Omnicam, as imagens podem ser adquiridas em movimento e sem utilização de agente de contraste (Sirona, 2018).

Após o escaneamento, um modelo virtual é criado em uma imagem 3D, para que seja realizado o planejamento virtual da restauração utilizando parâmetros estabelecidos pelo sistema conforme a definição anatômica da peça. Concluído o planejamento, a unidade fresadora prepara a restauração utilizando o material escolhido (Liu et al., 2008). As fresadoras mais utilizadas são a CEREC 3 Milling Unit, de modelo compacto e baixo custo, porém mais lenta e ruidosa, e a unidade CEREC MC $\mathrm{XL}$, introduzida no mercado posteriormente, e com aprimoramento em termos de velocidade de usinagem, reduzido nível de ruído, maior precisão de desgaste e simplicidade de operação, entretanto com custo mais elevado (Sirona, 2018).

Com o sistema CEREC para dentistas, toda a etapa laboratorial pode ser desenvolvida dentro do próprio consultório odontológico, apresentando maior articulação do passo-a-passo e conforto ao paciente durante o tratamento (Beuer et al.,2008). Diante disso, este artigo busca relatar um caso clínico odontológico realizado por meio do sistema CEREC.

\section{Metodologia}

O presente estudo remete-se à um relato de caso clínico, caracterizando-se em um estudo descritivo, respeitando os conceitos dos princípios éticos, a Declaração de Helsinque e após assinatura do Termo de Consentimento Livre e Esclarecido (TCLE) pelo responsável.

\section{Relato de Caso Clínico}

Paciente do sexo masculino, 22 anos de idade, procurou atendimento relatando ter feito uma restauração provisória no molar e fora encaminhado para realizar uma restauração definitiva em cerâmica. Durante a avaliação clínica e radiográfica, observou-se restauração provisória de ionômero de vidro no dente 46, de média profundidade e sem sintomatologia dolorosa (Figura 1).

Figura 1. Situação inicial: Restauração insatisfatória do dente 46.

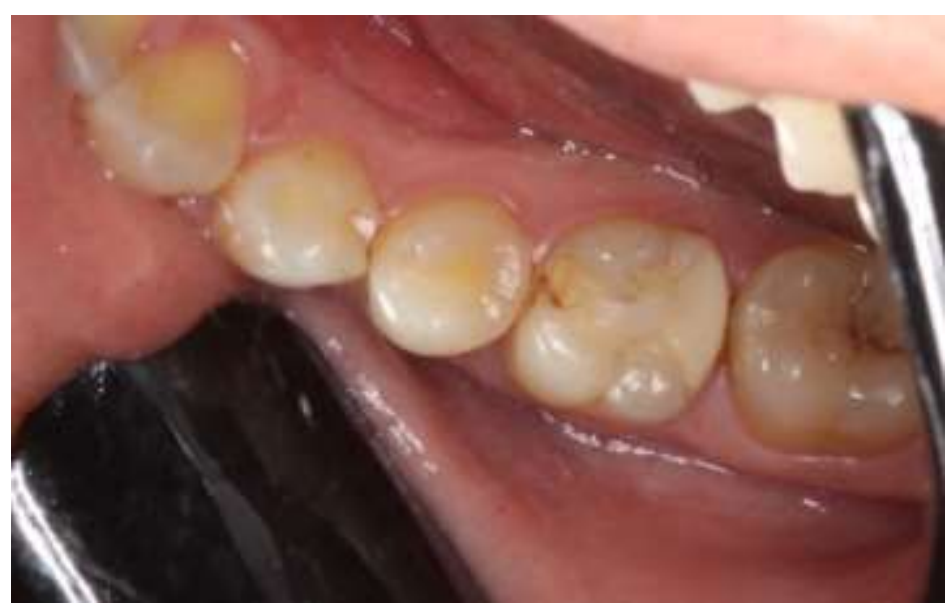

Fonte: Elaboração própria. 
Após exames iniciais, foi realizada a aferição da cor do dente através da escala Vita Clássica (R), o que definiu o bloco pré-fabricado CEREC Blocs C PC (Sirona Dental System, Suíça) de cerâmica feldspática de alta translucidez de cor A2. Em seguida foi realizada a remoção da restauração insatisfatória em ionômero de vidro por meio de uma fresa 1012 (KG Sorensen Indústria e Comércio LTDA, Brasil) e seguido de preparo de inlay do tipo MOD (mesio-ocluso-distal) à nível gengival na distal e com término em esmalte utilizando a fresa 2135 (KG Sorensen Indústria e Comércio LTDA, Brasil), arredondando todos os ângulos e tornando todo o preparo expulsivo (Figura 2). Em seguida, foi colocado o fio de afastamento gengival de espessura \#000 da Ultrapack (Ultradent ${ }^{\circledR}$, EUA) com auxílio de espátula para inserção Fischer’s Ultrapak Packer (Ultradent ${ }^{\circledR}$, EUA) no término distal (Figura 2).

Figura 2. Preparo MOD com fio de delicado afastamento gengival inserido na distal do dente 46 para melhor visualização do término em esmalte.

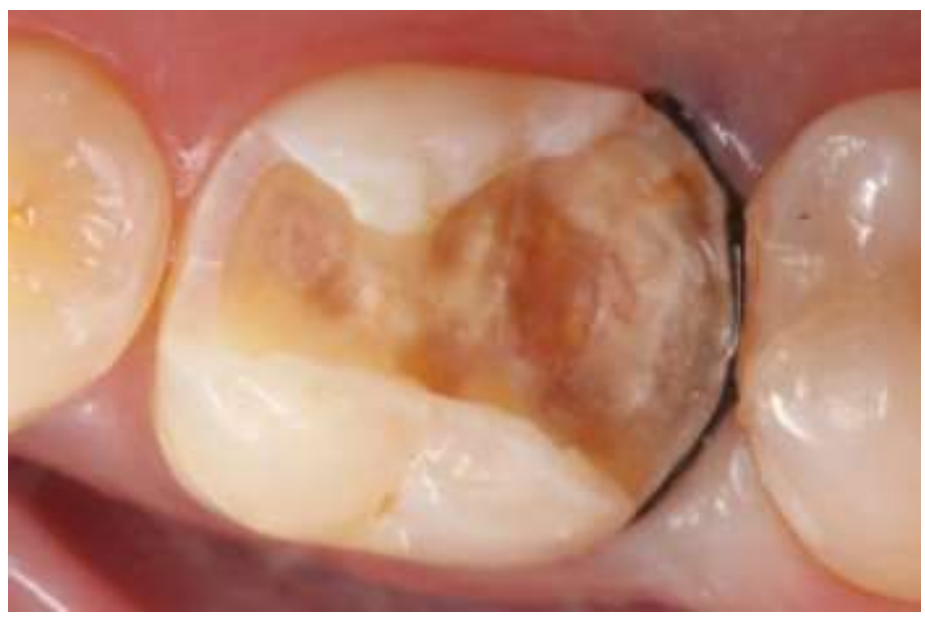

Fonte: Elaboração própria.

Então o trabalho em fluxo digital foi iniciado utilizando o CEREC Omnicam (Sirona Dental System, Suíça), que funciona como uma câmera filmadora para escaneamento. Foi colocado a câmera sobre a superfície dental e a digitalização iniciada automaticamente. Em seguida foi realizada a fase de escaneamento intraoral dos dentes 43 ao 47. Para isso, a área do preparo precisou estar devidamente seca e a câmera foi movida sobre os dentes do paciente em um único movimento de vestibular para palatina, caminhando da porção posterior para anterior da arcada. Os dados foram gerados automaticamente em um modelo 3D (Figura 3). Foi possível ainda interromper e retomar a digitalização a qualquer momento. Logo após, os dentes antagonistas à área correspondente ao preparo efetuado foram secos e escaneados, realizando o mesmo procedimento nas faces vestibulares da área correspondente com o paciente ocluindo de forma habitual para obtenção da relação de mordida (Figura 3). 
Figura 3. Escaneamento do dente preparado e da relação de mordida.
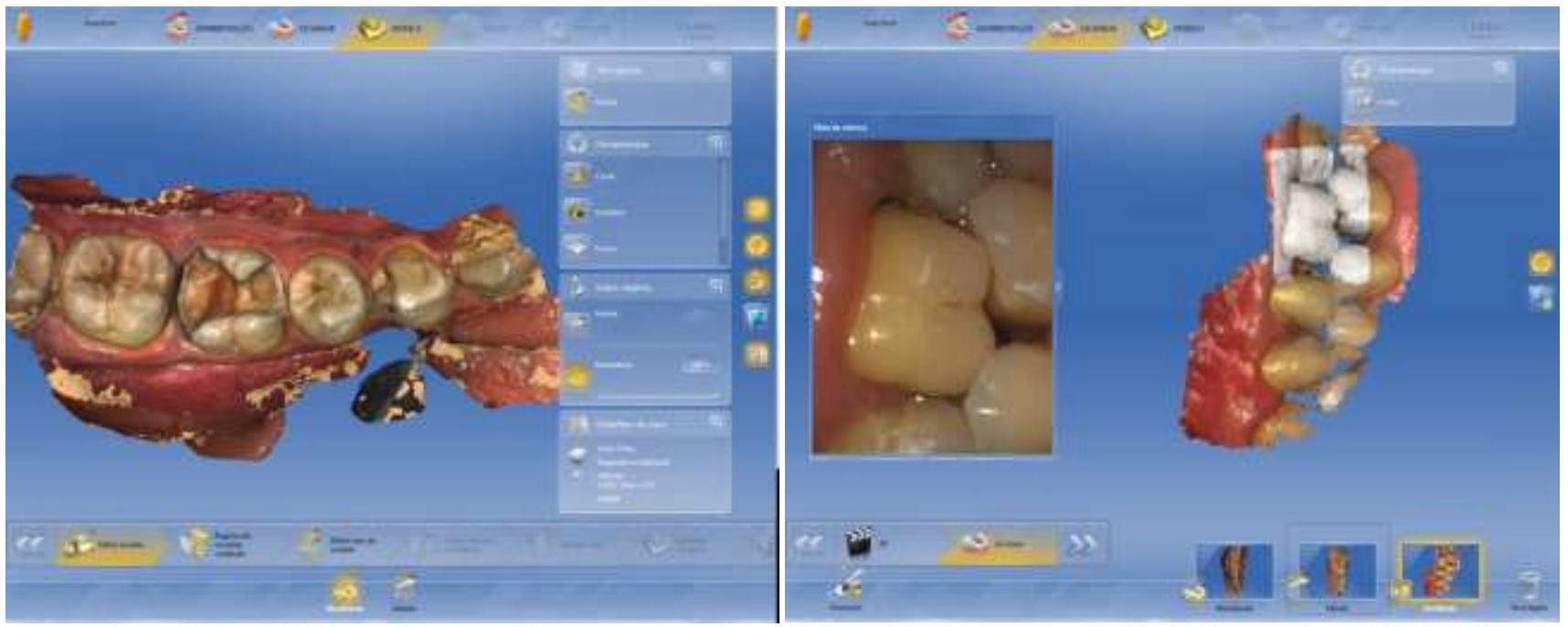

Fonte: Elaboração própria.

Em seguida, o software realizou dois traçados (vertical e horizontal) para que o modelo fosse movido e articulado digitalmente de acordo com a linha média e plano oclusal. Na sequência, foi realizada a delimitação do preparo ao longo de todo o dente utilizando a ferramenta de demarcar margem, partindo de um ponto inicial aleatório no término e fechando o ciclo no mesmo ponto rodeando-o no cavosuperficial (Figura 4). Foi definido também nessa fase o eixo de inserção da peça com uma seta acima do preparo que pôde ser manipulada em qualquer direção que torne esse eixo mais confortável para o encaixe da peça, além do software deixar visível caso o preparo esteja retentivo em alguma das faces. Após toda fase de delimitação e eixo de inserção, se estabeleceu o posicionamento do dente na arcada em 3D: verificando dimensão e inclinação mesio-distal e vestibulopalatina, além da altura do dente para que o programa calcule uma proposta de restauração individual (Figura 4).

Figura 4. Definição do eixo de inserção da peça e posicionamento do dente em relação à arcada dentária.

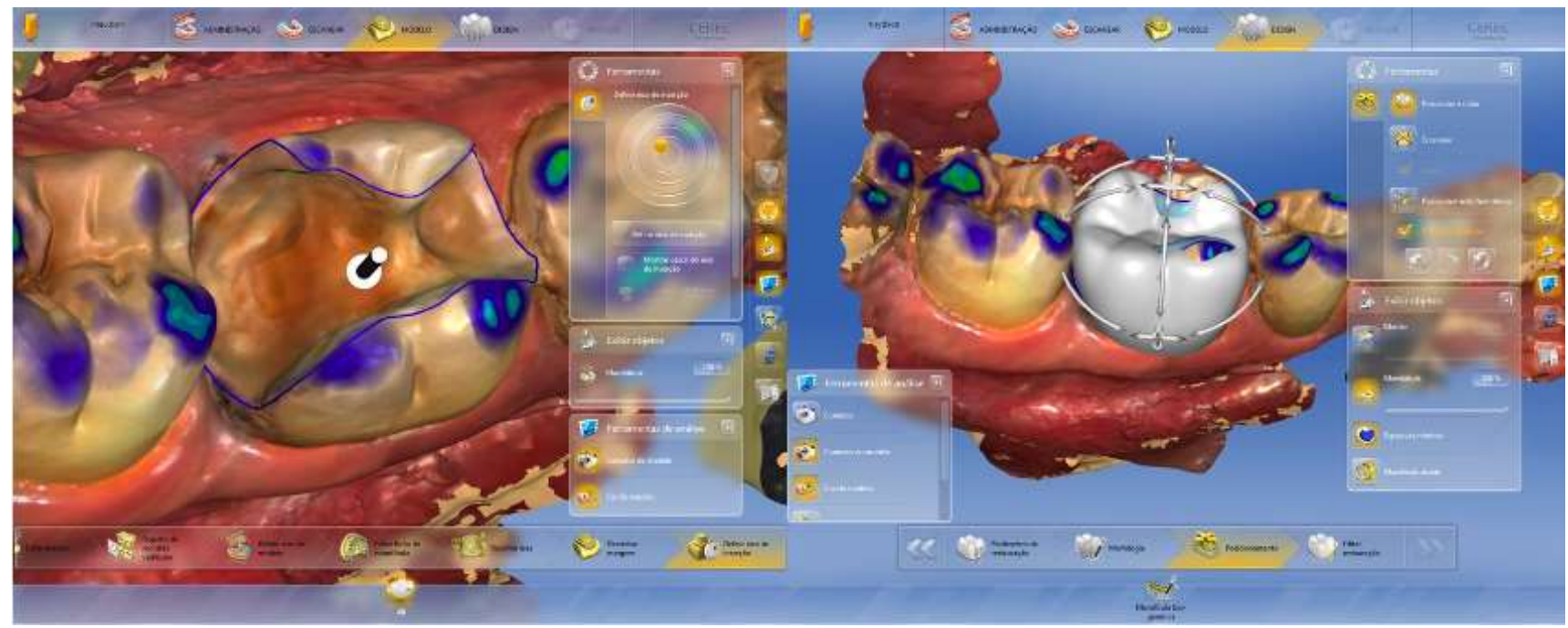

Fonte: Elaboração própria.

Assim, foi gerada a restauração indireta para ajuste e individualização através das ferramentas de anatomização que pode ser de forma circular ou anatômica. Na forma circular, foi apresentada uma seta sem forma definida para aumento de volume. Já na forma anatômica, a seta consegue manipular partes como cristas, cúspides e sulcos de forma íntegra e seguindo a 
anatomia pré estabelecida. (Figura 5). Foi assegurada a previsibilidade quanto ao ponto de contato proximal e oclusal. Em toda a região do dente, observou-se cores que são referentes às forças de contatos exercidos: o vermelho manifesta contato intenso, o amarelo contato forte, o verde ponto de contato convencional, o azul marinho de contatos leves. Na restauração em questão, o verde apareceu nos contatos proximais mesial e distal indicando o encaixe com toque suave e o azul escuro sinalizou o leve toque oclusal no dente antagonista (Figura 5).

Figura 5. Restauração gerada pelo software pronta para individualização dos ajustes e pontos de contatos previamente planejados.

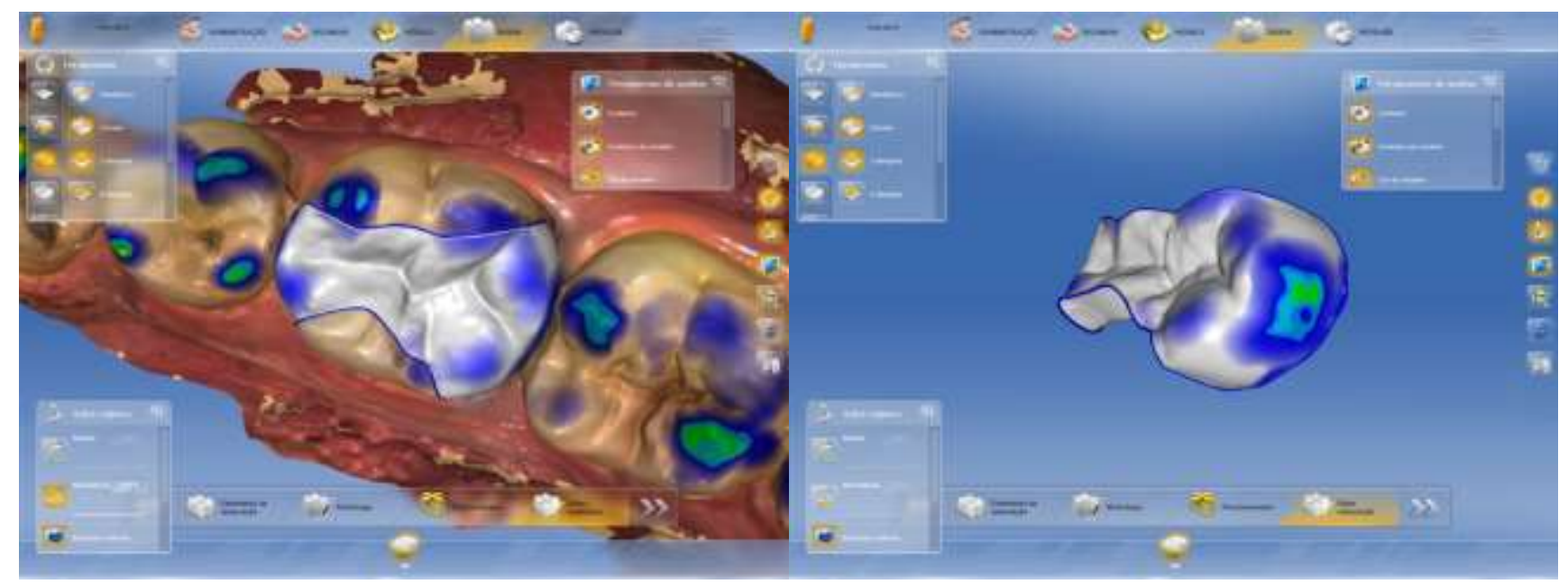

Fonte: Elaboração própria.

Depois do fluxo digital terminado e a restauração devidamente desenhada, o trabalho foi exportado para a unidade fresadora CEREC MC XL (Sirona Dental System, Suíça) para confecção da restauração indireta com o bloco pré-selecionado, iniciando o processo de fresagem com duração de 8 minutos e entrega da restauração pronta (Figura 6).

Figura 6. bloco fresado para a restauração indireta.

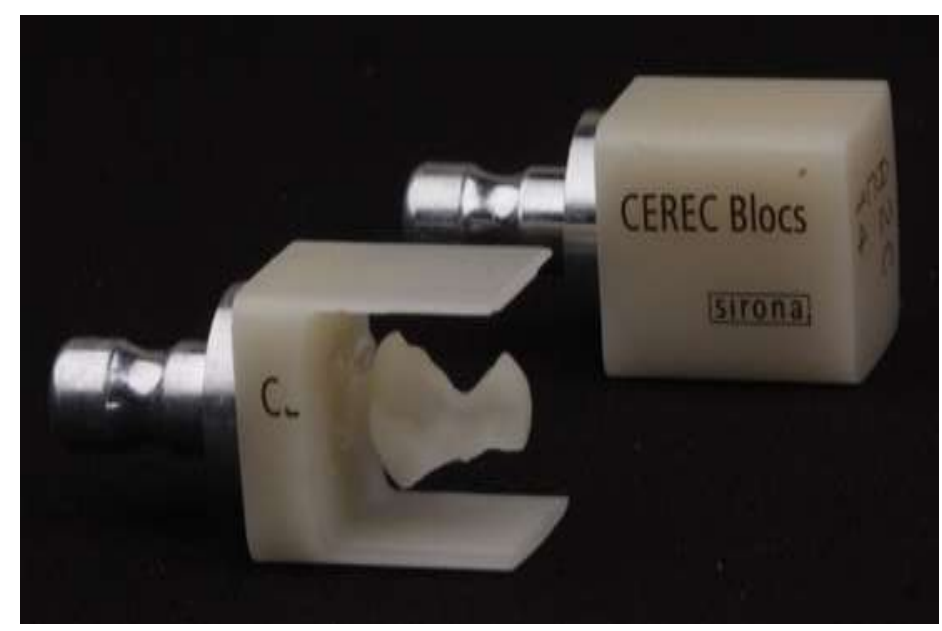

Fonte: Elaboração própria.

Após realizado o acabamento e polimento utilizando pontas de borrachas polidoras de cerâmica EVE Diapol azul, rosa e cinza (Ernst Vetter, Alemanha). Iniciou-se o desgaste com a EVE Diapol L26Dg azul para acabamento grosso, seguido da EVE 
Diapol R17Dmf rosa para acabamento médio e finalizou-se com a EVE Diapol L26D cinza para alto brilho e chegou-se ao brilho final (Figura 7).

Figura 7. Cerâmica feldspática após o polimento.

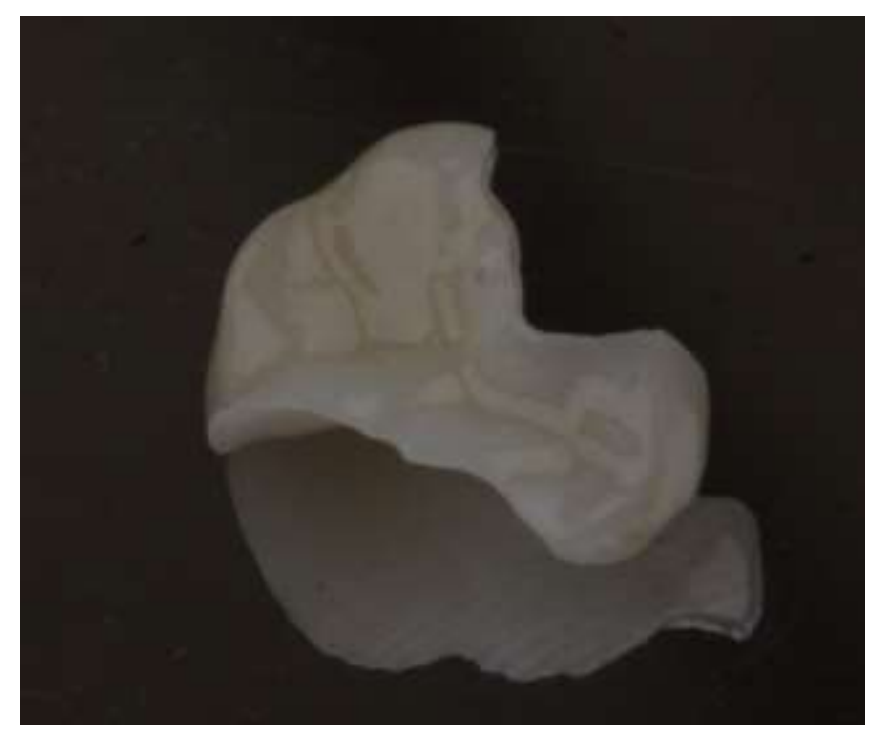

Fonte: Elaboração própria.

Na mesma sessão clínica, foi efetuada a prova da cerâmica no preparo em boca. Logo após, foi realizado o condicionamento da peça com ácido fluorídrico $10 \%$ Condac porcelana ( $\mathrm{FGM}^{\circledast}$ Produtos Odontológicos, Brasil) na face interna por 90 segundos seguidos de lavagem com jatos de água contínuos por 2 minutos para total remoção do ácido e secagem de superfície. Com o auxílio do aplicador Cavibrush (FGM ${ }^{\circledR}$ Produtos Odontológicos, Brasil), aplicou-se silano Prosil (FGM Produtos Odontológicos, Brasil) com movimento de fricção por 1 minuto na parte condicionada da peça, seguido pelo sistema adesivo universal Ambar APS (FGM ${ }^{\circledR}$ Produtos Odontológicos, Brasil). Logo após, foi realizado isolamento absoluto e profilaxia do preparo para limpeza com pedra pomes e água. Em seguida, iniciou-se o condicionamento do preparo com ácido fosfórico a $37 \%$ Condac (FGM ${ }^{\circledR}$ Produtos Odontológicos, Brasil) por 30 segundos em esmalte e 15 segundos em dentina, seguidos de lavagem pelo mesmo tempo de aplicação, para secagem e uso do sistema adesivo Ambar APS (FGM ${ }^{\circledR}$ Produtos Odontológicos, Brasil), jatos de ar à distância por 5 segundos e 40 segundos de ativação com fotopolimerizador à base de luz LED VALO ${ }^{\circledR}$ Cordless (Ultradent ${ }^{\circledR}$ Products Inc, Brasil).

A cimentação foi realizada com cimento resinoso dual cor A2 Allcem Dual (FGM ${ }^{\circledR}$ Produtos Odontológicos, Brasil). Este foi colocado sobre a superfície interna do dente preparado e da cerâmica tratada, e, logo posicionado sobre a superfície dental (Figura 24). Após o posicionamento exato, foi ativada a fotopolimerização passivamente por 3 segundos iniciais, sendo, então, retirados os excessos marginais, seguido pela polimerização das faces oclusal, palatina e vestibular, 40 segundos cada. Depois foi retirado o isolamento absoluto e testado oclusão com o auxílio da pinça Müller e papel carbono Accu Film ${ }^{\circledR}$ II $12 \mu \mathrm{m}$ (Parkell ${ }^{\circledR}$, USA) na superfície oclusal do dente em questão (Figura 8). Foi passado ao paciente as orientações de higiene para sucesso e conservação do procedimento restaurado, além de retorno frequente ao dentista. 
Figura 8. Cerâmica cimentada e contatos oclusais demarcados.

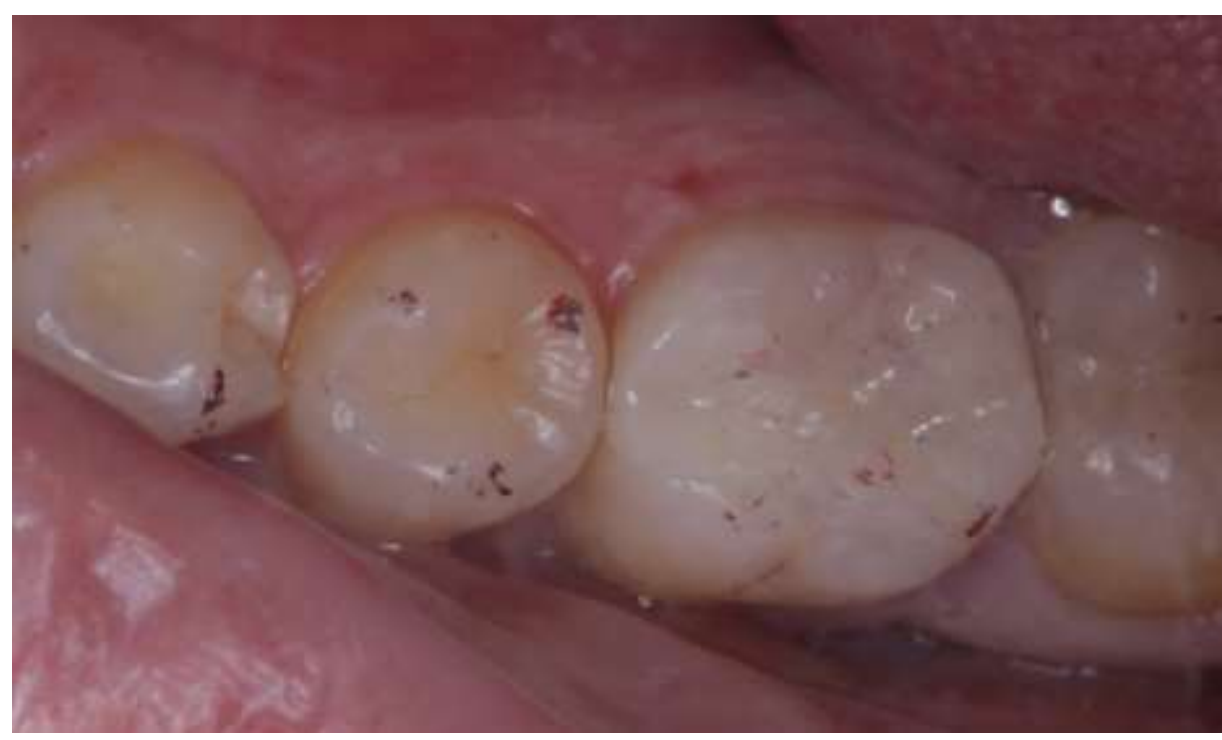

Fonte: Elaboração própria.

\section{Discussão}

$\mathrm{O}$ uso de restaurações indiretas de cerâmica no restabelecimento funcional e estético do sorriso em casos de grande perda de estrutura dental já está consagrado através de estudos científicos (Moreno et al., 2021). A existência da tecnologia CAD/CAM já possibilitou diversos estudos laboratoriais e clínicos analisando fatores que comumente são avaliados e comparados ao método tradicional de confecção das restaurações indiretas, como a qualidade e homogeneidade do material, adaptação marginal, controle da espessura da linha de cimento, longevidade e sucesso clínico das restaurações e estes corroboram com a previsibilidade, praticidade e qualidade do sistema CEREC (Cassiano et al., 2021).

As cerâmicas fresadas no sistema CEREC 3 tem consideráveis benefícios pois, com seu rígido controle de qualidade quanto à confecção de blocos pré-fabricados, assegura-se uma maior homogeneidade quando comparado às outras técnicas de elaboração de restaurações de cerâmica individuais em laboratórios, que dependem da habilidade dos técnicos em prótese dental (Kunzelmann et al., 2001). Tais restaurações pré-fabricadas têm propriedades mecânicas similares ou superiores aos fabricados convencionalmente e são mais resistentes ao desgaste, além de que, desgastam menos o esmalte do dente antagonista (Al-hiyasat et al., 1999).

As cerâmicas feldspáticas vêm sendo utilizadas na odontologia devido às suas propriedades estéticas, ou seja, de se assemelharem a estrutura natural do dente através de características ópticas e de translucidez além de serem biocompatíveis (Hilgert et al., 2005). Por outro lado, é um material friável, não suportando deformação plástica, no entanto, as técnicas adesivas e de cimentação melhoraram significativamente a resistência à fratura do material (Fasbinder, et al., 2013).

Estudos demonstraram que é possível alcançar excelentes níveis de adaptação das restaurações com o sistema CEREC 3 (Hilgert et al., 2005; Akbar et al., 2006; Mormann et al., 2000). Foi comparado, por diversos autores, a precisão de adaptação marginal quanto à diversos designs de término do preparo: as restaurações que foram confeccionadas no sistema CEREC 3 não tiveram diferenças significativas quanto ao tipo de término utilizado e todas apresentavam valores clinicamente aceitáveis e comparáveis aos valores de peças confeccionadas pelo método tradicional (Akbar et al., 2006; Mormann et al., 2000). Nesse sentido, durante a execução do caso clínico, necessitou-se de atenção e habilidade por parte do operador para perfeita delimitação do término da restauração para que esta consiga desenvolver melhor grau de adaptação marginal. 
Os valores médios de espessura de cimento são próximos a $50 \mu \mathrm{m}$ nas margens e ao redor de $110 \mu \mathrm{m}$ na interface interna (Fasbinder et al., 2013; Poticny et al.,2010). Foi observado na restauração produzida pelo sistema CEREC 3, que esta foi devidamente mensurada e admitiu-se uma pequena área de cimento sujeita ao desgaste pela abrasão e espessura propícia para evitar acenturados efeitos do estresse causado pela contração de polimerização, como explicito nos estudos. O sistema CEREC possui taxas de sucesso das restaurações por volta de $97 \%$ após cinco anos e $90 \%$ após 10 anos, demonstrando que o longo prazo de conservação e efetividade clínica do sistema CEREC é uma opção válida para restaurações deste tipo (Fasbinder et al., 2013).

Algumas desvantagens são descritas nos estudos sobre o sistema: as dificuldades de sensibilidade técnica do procedimento de captação óptica dos dentes preparados e a necessidade de equipamentos de alto custo (Kim et al., 2015; Chain et al., 2000; Christensen et al., 2008). Estas dificuldades quanto à captação das imagens utilizando a Omnicam foram superadas pelo operador neste estudo de caso, necessitando de uma curva de aprendizado quanto à manipulação do scanner intraoral. No entanto, são propostos cursos de qualificação profissional pelo próprio fabricante para o correto manuseio de todo o sistema além de que existe ainda a possibilidade de o dentista adquirir apenas o scanner intraoral e o software de planejamento, onde os elementos do preparo são enviados eletronicamente para um laboratório que possua o sistema CEREC para laboratório (inLab) visando a produção da peça protética (Mormann et al., 2000, Chain et al., 2000).

Outra dificuldade encontrada pelos estudos é de como inserir a conceituação do novo sistema na clínica diária tendo em vista o alto custo inicial dos sistemas e acessórios completos. Ainda assim, é preciso avaliar que, apesar que cerâmicas confeccionadas no sistema CEREC possuem um alto investimento inicial, o custo-benefício é superior comparado com os fabricados em cerâmica por um laboratório, quando avaliado a médio prazo, onde o dentista tem uma redução expressiva nas taxas de laboratório, tendo um bom retorno sobre investimento (Poticny et al., 2010; Christensen 2008).

É bastante notório que a odontologia está em constante mudança através da inclusão de inovadoras tecnologias e o caminho destas é tornar-se menos oneroso. Em pouco tempo, o uso de sistemas CAD/CAM como o CEREC fará parte não só do cotidiano de uma pequena fração de profissionais, mas tornar-se-a protocolo padrão em grande parte dos consultórios e laboratórios de prótese dentária por exigência de mercado, onde irá diminuir os custos finais para dentistas, laboratórios e pacientes (Akbar et al., 2006)

\section{Conclusão}

O sistema CEREC para dentistas detém consequências positivas e comprovadas no caso clínico executado, dispondo como resultado restaurações de excelente qualidade sendo feitas dentro do próprio consultório, apresentando grande benefício para o paciente e para a prática diária do dentista. Além disso, apresenta um retorno financeiro vantajoso em médio prazo e a disposição é que seu aproveitamento se torne integrante não só do cotidiano de uma pequena parcela dos cirurgiões-dentistas, mas sim protocolo padrão na maioria dos consultórios. Desta forma, sugere-se que ensaios clínicos randomizados com acompanhamento ao longo dos anos sejam realizados para corroborar com os resultados obtidos neste relato de caso clínico.

\section{Referências}

Akbar, J. H., Petrie, C. S., Walker, M. P., Williams, K., \& Eick, J. D. (2006). Marginal adaptation of Cerec 3 CAD/CAM composite crowns using two different finish line preparation designs. Journal of Prosthodontics: Implant, Esthetic and Reconstructive Dentistry, 15(3), 155-163.

Beuer, F., Schweiger, J., \& Edelhoff, D. (2008). Digital dentistry: an overview of recent developments for CAD/CAM generated restorations. British dental journal, 204(9), 505-511.

Cassiano, C. K. P., Dias, S. C., Rigolin, F., de Oliveira Mussel, R. L., dos Santos, L. M., \& Tiossi, R. (2021). Shear bond strength between resin cement and lithium disilicate ceramics after intrinsic staining. Research, Society and Development, 10(3).

Chain, M. C., Arcari, G. M., \& Lopes, G. C. (2000). Restauraçöes cerâmicas estéticas e próteses livres de metal: as novas alternativas possibilitadas pelas novas porcelanas. RGO (Porto Alegre), 67-70. 
Research, Society and Development, v. 10, n. 6, e54310616142, 2021

(CC BY 4.0) | ISSN 2525-3409 | DOI: http://dx.doi.org/10.33448/rsd-v10i6.16142

Christensen, G. J. (2008). Successful use of in-office CAD/CAM in a typical practice. The Journal of the American Dental Association, 139(9), 1257-1260.

Dukic, W., Dukic, O. L., Milardovic, S., \& Delija, B. (2010). Clinical evaluation of indirect composite restorations at baseline and 36 months after placement. Operative dentistry, 35(2), 156-164.

Duret, F., Blouin, J. L., \& Duret, B. (1988). CAD-CAM in dentistry. The Journal of the American Dental Association, 117(6), 715-720.

Fasbinder, D. J. (2010). The CEREC system: 25 years of chairside CAD/CAM dentistry. The Journal of the American Dental Association, 141, 3S-4S.

Fasbinder, D. J., Neiva, G. F., Dennison, J. B., \& Heys, D. R. (2013). Clinical Performance of CAD/CAM-Generated Composite Inlays After 10 Years. Journal of Cosmetic Dentistry, 28(4).

Hilgert, L. A., Calazans, A., \& Baratieri, L. N. (2005). Restaurações CAD/CAM: o sistema CEREC 3. Clín. int. j. braz. dent, 198-209.

Keshvad, A., Hooshmand, T., Asefzadeh, F., Khalilinejad, F., Alihemmati, M., \& Van Noort, R. (2011). Marginal gap, internal fit, and fracture load of leucitereinforced ceramic inlays fabricated by CEREC inLab and hot-pressed techniques. Journal of Prosthodontics: Implant, Esthetic and Reconstructive Dentistry, 20(7), 535-540.

Kim, J. H., Kim, K. B., Kim, S. H., Kim, W. C., Kim, H. Y., \& Kim, J. H. (2015). Quantitative evaluation of common errors in digital impression obtained by using an LED blue light in-office CAD/CAM system. Quintessence Int, 46(5), 401-7.

Kunzelmann, K. H., Jelen, B., Mehl, A., \& Hickel, R. (2001). Wear evaluation of MZ100 compared to ceramic CAD/CAM materials. International journal of computerized dentistry, 4(3), 171-184.

Al-Hiyasat, A. S., Saunders, W. P., \& Smith, G. M. (1999). Three-body wear associated with three ceramics and enamel. The Journal of prosthetic dentistry, 82(4), 476-481.

Liu, P. R., \& Essig, M. E. (2008). Panorama of dental CAD/CAM restorative systems. Compendium of continuing education in dentistry (Jamesburg, NJ: 1995), 29(8), 482-484.

Miyazaki, T., Hotta, Y., Kunii, J., Kuriyama, S., \& Tamaki, Y. (2009). A review of dental CAD/CAM: current status and future perspectives from 20 years of experience. Dental materials journal, 28(1), 44-56.

Moörmann, W. H. (2006). The evolution of the CEREC system. The Journal of the American Dental Association, 137, 7S-13S.

Moörmann, W. H., \& Bindl, A. (2000). The Cerec 3--A quantum leap for computer-aided restorations: Initial clinical results. Quintessence international, 31(10).

Moreno, J. M. L., Oliveira, D. de, Sales, P. S. M., Marchiolli, C. L., Tenório, B. M., Barion, A. R., Toscano, R. A., Rocha, E. P., \& Assunção, W. G. (2021). Os índices de trincas e fraturas em laminados cerâmicos são por razões oclusais? Uma revisão sistemática. Research, Society and Development, 10(5), e7810514620.

Nakamura, T., Dei, N., Kojima, T., \& Wakabayashi, K. (2003). Marginal and internal fit of Cerec 3 CAD/CAM all-ceramic crowns. International Journal of Prosthodontics, 16(3).

Poticny, D. J., \& Klim, J. (2010). CAD/CAM in-office technology: innovations after 25 years for predictable, esthetic outcomes. The journal of the American dental association, 141, 5S-9S.

Sirona the dental company. Empresa Sirona. [2013]. 〈http://www.sirona.com.br/br/empresa/about-sirona〉. 